\title{
DE L'INFLUENCE DES BARRAGES DE RETENUE SUR LES PEUPLEMENTS SALMONICOLES
}

\author{
par \\ Roger BACHELIER \\ Ingénieur des Eaux et Forêts
}

Dans un article sur l'Intérêt piscicole des retenues hydroélectriques, publié dans le Bulletin Français de Pisciculture d'Avril-Juin 1950, j'avais indiqué que non seulement un barrage de retenue construit dans une rivière à truites condamnait à la disparition les lignées de salmonidés anadromes qui pouvaient frayer à son amont antérieurement à sa construction, mais encore que la retenue d'eau elle-même pouvait devenir impropre à la vie de tout salmonide en raison de la diminution de sa teneur en oxygène en été. Cet appauvrissement en oxygène était expliqué par le double effet de la fermentation des dépôts dans le fond et de l'insolation de la surface en été.

Cependant, les observations faites sur l'évolution des peuplements de certaines retenues depuis une dizaine d'années et sur les teneurs en oxygène des eaux de nombreuses retenues depuis deux ans, ont montré que la teneur en oxygène en été pouvait n'être pas seule en cause, et que celle-ci dépendait non seulement de l'altitude, mais de plusieurs autres facteurs tels que la situation, les dimensions et l'utilisation de la retenue. Il y a lieu de préciser que toutes ces observations ont été faites dans des retenues situées en terrain primaire très pauvre et à moyenne altitude (inférieure à $800 \mathrm{~m}$. et en majorité entre 5 et $700 \mathrm{~m}$.).

\section{LES SALMONIDÉS ET LA TENEUR EN OXYGENE}

La classification des cours d'eau en $1^{\text {re }}$ et $2^{\text {e }}$ catégorie les distingue suivant qu'ils sont peuplés plus ou moins abondamment ou pas du tout en salmonides. Cependant, les Truites qui peuplent en minorité la partie amont de la $2^{e}$ catégorie et théoriquement en majorité la partie aval de la $1^{\text {re }}$ catégorie, sur une longueur excessivement variable, ne sont jamais que des truites adultes. Et cela, même si ces Truites doivent pondre dans ce secteur faute de pouvoir remonter en raison de barrages infranchịsables.

Indépendamment de l'absence de frayère due généralement à une vitesse insuffisante du courant, deux explications de l'absence d'alevins 
de salmonides dans ce secteur intermédiaire peuvent ètre envisagées : soit la concurrence des poissons blancs, soit l'insuffisance de la teneur en oxygène de ces eaux en été.

Les constatations faites dans les retenues hydrauliques, dont les eaux mortes et les fonds vaseux ne peuvent permettre la reproduction des Truites, prouvent que cette deuxième explication peut être suffisante.

En effet, ce n'est qu'exceptionnellement que la température des eaux baignant des frayères fertiles dépasse une vingtaine de degrés; température à laquelle l'eau agité contient environ $6,4 \mathrm{~cm}^{3}$ d'oxygène au litre ; or cette teneur en oxygène est loin d'être atteinte en été, même en surface dans plusieurs retenues du Massif Central peuplées encore exclusivement de Truites.

Au cours de ces deux années de relevés des teneurs en oxygène, celles-ci, dans des retenues où les Truites sont habituelles, n'ont souvent pas dépassé $5,8 \mathrm{~cm}^{3}$ au litre en été, et soit sur une assez grande profondeur, soit seulement en surface, ce qui correspond à la teneur en oxygène d'une eau agitée à une température de $26^{\circ}$.

En conséquence, on peut chercher à constituer un peuplement de Truites communes dans les retenues où la teneur en oxygène en été ne s'abaisse pas au-dessous de $5,8 \mathrm{~cm}^{3}$, au moins à une certaine profondeur, en déversant des truitelles en automne. A cette époque la teneur en oxygène a déjà augmenté de 1 à $2 \mathrm{~cm}^{3}$ par litre, si bien que ces truitelles pourront survivre et devenir moins exigeantes en oxygène avant la régression de la teneur de ce gaz au cours de l'été suivant.

Il est probable que la limite inférieure de la teneur en oxygène pour la Truite arc-en-ciel serait encore plus faible, peut-être $5,6 \mathrm{~cm}^{3}$ correspondant à la teneur d'une eau agité à 29०. Aucune observation n'a encore été faite à ce sujet, et, en fait, une telle observation ne pourrait avoir lieu qu'en constituant des peuplements mixtes dans des retenues dont la teneur estivale en oxygène risquerait, les années chaudes, de descendre au-desssous de $5,8 \mathrm{~cm}^{3}$ dans la quasi-totalité de leur volume.

\section{LA TENEUR EN OXYGËNE DES EAUX DES RETENUES}

Il avait été avancé, dès 1950, que les eaux des retenues étaient appauvries en oxygène, en été, par la fermentation des dépôts et par l'échauffement de leur surface du fait de l'ensoleillement,

Ces deux facteurs, incontestablement certains, sont très variables suivant la situation, les dimensions et l'utilisation des retenues; et il est apparu que la décomposition de la végétation dans une nouvelle retenue peut provoquer temporairement un troisième facteur d'appauvrissement en oxygène. 


\section{Influence de la situation}

Indépendamment de l'altitude, qui est favorable à une plus forte teneur en oxygène en raison de la moindre élévation de la température ambiante, une retenue située à l'aval d'une grande retenue annuelle peut être plus oxygénée que cette dernière en saison chaude. Cette réoxygénation de l'eau à basse altitude, s'explique facilement du fait qu'il s'agit de l'eau de profondeur de la retenue amont, relativement fraîche en été, qui se sature d'oxygène dès son contact avec l'air à la sortie des turbines. En outre, la majorité des matières en suspension s'étant déposées dans la retenue amont, d'une part il peut n'y avoir que peu de vase dans la retenue aval, et d'autre part l'eau de cette dernière est plus transparente et donc plus favorable à la microflore, qui, par l'assimilation chlorophyllienne, peut libérer l'oxygène du gaz carbonique produit par la microfaune.

Un exemple tout à fait remarquable de ce rafraîchissement de l'eau en été,auquel correspond d'ailleurs un réchauffement en hiver, est donné par les grandes retenues qui s'échelonnent sur la Dordogne.

Jadis, la limite entre la $1^{\text {re }}$ et la $2^{\mathbf{e}}$ catégorie dans la Dordogne était située au voisinage de Bort-les-Orgues à une altitude de 430 mètres.

Or, depuis qu'il a été créé 3 retenues successives totalisant 300 millions de mètres cubes sur 80 kilomètres de longueur à l'aval et une retenue de 400 millions de mètres cubes longue de 20 kilomètres à l'amont de Bort, les Truites ont fait leur apparition à 100 kilomètres à l'aval de l'ancienne limite de la $1^{\text {re }}$ catégorie à une altitude de 140 mètres seulement. D'ailleurs, depuis 5 ans que les températures de l'eau sont relevées à une vingtaine de kilométres à l'aval de la dernière chute, cellesci ne se sont abaissées qu'une fois à $2^{\circ}$ et n'ont jamais dépassé $21^{\circ}$.

\section{Influence des dimensions}

La teneur en oxy ène des eaux d'une retenue dépend des dimensions de celle-ci, dans la mesure où ces dimensions influent sur la température. En effet, une retenue très peu profonde s'échauffera beaucoup plus par ensoleillement qu'une retenue profonde, d'autant plus que cette dernière a normalement des rives très escarpées qui la cachent plus ou moins du soleil.

Au surplus, dans une retenue peu profonde, la proportion du matelas anaréobie du fond provoqué par la vase est beaucoup plus grande.

\section{Influence du mode d'utilisation}

Cependant, ces facteurs de désoxygénation des retenues peu profondes peuvent être compensés par une plus grande vitesse d'écoulement des eaux.

En raison des conditions topographiques et géologiques, certains barrages à destination essentielle de prise d'eau ont été surélevés pour 
constituer acces oirement des petites retenues temporaires, dans ce cas la vitesse d'écoulement de l'eau y est telle qu'il n'y a pas de désoxygénation sensible.

Un autre cas où les facteurs de désoxygénation peuvent être très réduits $d u$ fait du mode d'utilisation de la retenue est constitué par les retenues dont l'exploitation hydroélectrique comporte une vidange quasitotale chaque année. Du fait de cette exploitation, outre que les eaux sont renouvelées au moins chaque année, les vases découvertes pendant plusieurs mois sont oxydées par l'air et perdent leur pouvoir réducteur.

\section{Influence de la décomposition de la végétation noyée}

Des mortalités massives de Truites ont été constatées au cours de l'été 1935 dans la retenue de Sarrans, qui avait été mise en eau en 1933, et de même en août 1947 dans la retenue de Neuvic, qui avait été remplie pour la première fois en 1945 .

En outre, la retenue de Bort, dont il a été question ci-dessus et qui avait été mise en eau en 1951, a été moins riche en oxygène que la retenue du Chastang située à 80 kilomètres à son aval, en 1956, tandis qu'elle est redevenue plus riche en 1957.

Il semble donc qu'il y ait une brusque diminution de la teneur en oxygène dissous quelques années après la mise en eau d'une retenue hydroélectrique et que celle-ci, correspondant à la décomposition de la végétation noyée, puisse n'être que temporaire.

Il y a lieu de remarquer, d'ailleurs, que l'effet réducteur de la décomposition de la végétation doit jouer sur toute la surface de la retenue, quelle que soit la profondeur d'eau, tandis que l'effet réducteur de la fermentation des dépôts dépend de l'accumulation de ces dépôts généralement plus faible sur la périphérie de la retenue.

Cette différenciation des causes de l'appauvrissement de cette teneur en oxygène pourrait expliquer l'évolution de la répartition de la teneur en oxygène dans la retenue de Sarrans, où $M$. l'Inspecteur Général LARRIEU, alors Inspecteur Principal, avait procédé à des dosages de l'oxygène en 1936, dosages qui ont été renouvelés aux mêmes points en 1957.

La retenue de Sarrans, de 100 mètres de creux au plus profond et d'un millier d'hectares au plein à la cote $646 \mathrm{~N}$ G F, a été mise en eau au cours de l'automne 1933 pour alimenter une usine avec un débit au plus égal à 120 mètres cubes sec. Il s'agit donc d'une retenue annuelle à renouvellement très lent de l'eau.

Les relevés de température et de teneur en oxygène à 100 mètres et à 1.500 mètres ( Pont Treboul) en amont du barrage en 1936 et 1957 furent les suivants : 
100 mètres en amont du barrage.

\begin{tabular}{|c|c|c|c|c|c|c|}
\hline \multirow{2}{*}{ Profondeur } & \multicolumn{2}{|c|}{17.7 .36} & \multicolumn{2}{|c|}{29.9 .36} & \multicolumn{2}{|c|}{28.8 .57} \\
\hline & To & $0 \mathrm{~cm}^{3}$ & $\mathbf{T}^{\mathrm{o}}$ & $0 \mathrm{~cm}^{3}$ & To & $0 \mathrm{~cm}^{3}$ \\
\hline $\begin{array}{l}\text { Surface } \ldots \ldots \ldots \\
10 \text { mètres } \ldots \ldots \ldots \\
20 \text { mètres } \ldots \ldots \ldots \\
30 \text { mètres } \ldots \ldots \ldots \\
\mathbf{4 0} \text { mètres } \ldots \ldots \ldots \\
\mathbf{5 0} \text { mètres } \ldots \ldots \ldots \\
60 \text { mètres } \ldots \ldots \\
\mathbf{8 0} \text { mètres } \ldots \ldots\end{array}$ & $\begin{array}{r}18,2 \\
16,4 \\
11,8 \\
10,2 \\
8,9 \\
8 \\
7,6 \\
5,8\end{array}$ & $\begin{array}{l}5 \\
4,8 \\
3,5\end{array}$ & $\begin{array}{l}18 \\
17,8 \\
17 \\
16\end{array}$ & 2,5 & $\begin{array}{c}19,2 \\
16,8 \\
11 \\
7 \\
7\end{array}$ & $\begin{array}{c}5,5 \\
4,7 \\
4 \\
1,8 \\
0\end{array}$ \\
\hline
\end{tabular}

1.500 mètres en amont du barrage

\begin{tabular}{|c|c|c|c|c|}
\hline \multirow{2}{*}{ Profondeur } & \multicolumn{2}{|c|}{$11 / 7 / 36$} & \multicolumn{2}{|c|}{$28 / 8 / 57$} \\
\hline & $T^{\mathbf{o}}$ & $0 \mathrm{~cm}^{3}$ & To & $0 \mathrm{~cm}^{3}$ \\
\hline $\begin{array}{l}\text { Surface } \ldots \ldots \ldots \ldots \\
\mathbf{1 0} \text { mètres } \ldots \ldots \ldots \ldots \\
\mathbf{2 0} \text { mètres } \ldots \ldots \ldots \ldots \\
\mathbf{3 0} \text { metres } \ldots \ldots \ldots \ldots\end{array}$ & $\begin{array}{l}19,8 \\
17,1 \\
11,8 \\
10,4\end{array}$ & $\begin{array}{l}5,5 \\
4,9 \\
5 \\
4,9\end{array}$ & $\begin{array}{l}19,4 \\
18,3 \\
17,7 \\
13,8\end{array}$ & $\begin{array}{c}5,8 \\
4,8 \\
1,8 \\
0\end{array}$ \\
\hline
\end{tabular}

L'appauvrissement général de la teneur en oxygène en 1936 s'explique par la décomposition de la végétation noyée quelle que soit la profondeur d'eau, et la stratification ultérieure de cette teneur par l'absence d'écoulement de l'eau au-dessus des vases accumulées.

Quoiqu'il en soit, cette retenue de Sarrans, qui était devenue tout à fait impropre aux salmonides en 1936, doit dorénavant pouvoir leur convenir superficiellement dans la majorité de son étendue à l'amont.

\section{LA DESTINATION SALMONICOLE D'UNE RETENUE HYDROÉLECTRIQUE}

Lors de mon exposé sur l'intérêt piscicole des retenues hydroélectriques en 1950, j'avais cru pouvoir expliquer la raréfaction des Truites dans des retenuesoù elles vivaient parfaitement, par un défaut dans leur reproduction, celui-ci pouvant être dû, soit à la présence d'une chute' 
infranchissable inmédiatement à l'amont du remous de la retenue au moment de la migration reproductrice, soit à la submersion des pontes par l'eau morte de la retenue en remplissage après la fraye.

Cependant, ces raisons ne suffisent pas à expliquer la très grande régression des Truites dans de nombreuses retenues où, pourtant, il en subsiste toujours.

En fait, dans la majorité des retenues qui nous intéressent, comme à la limite des première et deuxième catégorie dans les cours d'eau la teneur en oxygène des eaux en été est suffisante pour les salmonides adultes, ou tout au moins d'une certaine taille, mais non pour les alevins qui doivent mourir par asphyxie sans laisser de trace dans ces grandes masses d'eau.

Cette explication est d'autant plus plausible que, contrairement aux Truites communes qui se laissent descendre surtout à l'état d'alevins lors des crues de printemps, les Truites de mer et les Saumons ne quittent leurs frayères d'origine qu'après un ou même deux étés pour affronter les eaux de deuxième catégorie, puis la mer.

En outre, il y a plusieurs retenues où les Truites ne cherchent pas à remonter les affluents pour frayer et lâchent leurs œufs dans la retenue même, à moins qu'elles ne soient stériles (ces dernières sont très facilement reconnaissables car elles sont argentées). Cette inaptitude quasi-totale de certains peuplements à remonter dans les ruisseaux pour frayer est-elle due à ce que le peuplement autochtone était sédentaire? Ce pourrait justement être le cas dans les secteurs de cours d'eau où sont établies certaines retenues.

La raréfaction des Truites dans les retenues peut donc être due à plusieurs causes autres que l'insuffisance de l'oxygène pour leur besoin respiratoire à l'état adulte :

- Inaccessibilité des frayères, (chute infranchissable à l'amont immédiat du remous).

- Submersion des frayères, (lorsque la retenue est à l'étiage en automne).

- Mortalité accidentelle des Truites par la décomposition des végétaux submergés.

- Mortalité au cours de leur premier été des alevins descendus dans la retenue au printemps.

- Inaptitude des Truites à s'échapper de la retenue pour frayer.

En conséquence, avant de renoncer à la destination salmonicole d'une retenue et $y$ introduire des poissons blancs qui constitueront toujours une concurrence pour les Truites, il importe de s'assurer, 4 ou 5 ans après la mise en eau totale de la retenue, que la teneur en oxygène ne s'y abaisse pas au-dessous de $5,8 \mathrm{~cm}^{3}$ en surface pendant le mois d'août, même si le peuplement naturel en Truites a régressé considérablement. 
Si la teneur en oxygène ne s'abaisse jamais au-dessous de $5,8 \mathrm{~cm}^{3}$ au moins en surface, il devra être procédé à des alevinages d'automne et, si possible, avec des Truites "dévalentes " pour employer le terme des pêcheurs à la ligne vis-à-vis des alevins de Truite arc-en-ciel qu'ils n'ont pas retrouvés en pêchant. Mais dévalentes seulement après leur premier été, ce qui doit être le fait habituel des Truites de mer et peut-être des Truites de lac. Reste à savoir si des Truites de mer peuvent s'acclimater dans une retenue, mais l'essai vaut d'être tenté.

La quantité de truitelles à déverser devrait être calculée suivant la capacité biogénique de la retenue et compte tenu de la concurrence créée par le peuplement préexistant.

La capacité biogénique d'une retenue hydroélectrique est très inférieure à celle de la rivière naturelle qu'elle a remplacée en raison de l'absence de végétation et souvent même de toute microflore faute d'oxygène ou de lumière à partir d'une certaine profondeur.

Tant qu'aucune végétation ne s'est installée sur les bords et si l'eau est très opaque ou pauvre en oxygène en profondeur, une retenue établie en terrain granitique ne pourra supporter un peuplement que de l'ordre d'une dizaine de kilos à l'hectare, si j'en juge par les vidanges de retenues auxquelles j'ai assisté.

Or, en fait, on ne pourra espérer l'installation d'une végétation marginale que dans certaines grandes retenues, construites primitivement pour constituer des retenues annuelles, et utilisées seulement pour des pointes journalières depuis la création de nouvelles grandes retenues annuelles à leur amont. En effet, ces retenues alimentant de basses chutes, le concessionnaire aurait grand avantage à ne pas en abaisser le niveau.

Par contre, il n'y a pas de possibilité de végétation marginale même pour les nombreuses retenues annuelles qui sont destinées à constituer des réserves d'eau pour la fin de l'été et le début de l'automne, jusqu'aux pluies, et qui, par conséquent, sont traitées comme des étangs. En effet, à la différence des étangs, même de ceux dont le remplissage est le plus aléatoire, le niveau de remplissage d'une retenue annuelle varie de plusieurs mètres d'une année à l'autre, et cela non seulement parce que sa profondeur est plus grande que celle d'un étang si grand soit-il, mais encore parce que, en année sèche, il est fait appel à sa réserve d'eau quelle que soit la saison. Il faudrait une succession d'une dizaine d'années humides pour que ces retenues soient utilisées vraiment comme des étangs et puissent être envahies par une végétation.

Les peuplements des retenues qui nous intéressent comportent habituellement : des Ablettes, des Chevaines et des Perches (introduites par les pêcheurs), c'est-à-dire les trois espèces qui se reproduisent malgré les variations de niveau des plans d'eau qui les abritent. Les Ablettes constituant un poisson-fourrage, ne sont sans doute pas concurrentes pour les Truites, mais il n'en est pas de mème pour les Chevaines et les Perches. Pour ces dernières, une observation faite dans la retenue fréquemment 
vidangée Lavaud-Gelade, laisserait supposer que la Perche naine peut constituer un danger mortel pour les grosses Truites. En effet, les Truites de 2 à 4 livres, qui constituaient la quasi totalité du peuplement de cette retenue en 1950, avaient presque totalement disparu lors de la vidange de 1953 où il fut récolté 2 tonnes de Perches naines et 1.200 Truites de 100 à 300 grammes au plus, sauf quelques rares individus plus gros.

Il ne semble donc pas qu'il soit possible de lutter contre la pullulation des Perches par des déversements de truitelles, et avant de tenter l'enrichissement en salmonidés d'une retenue contenant des Perches naines, il faudrait mieux couper tous les bois morts noyés à faible profondeur qui leur servent de frayères et même, si possible, piéger les rubans de fraie des Perches au moyen de branchages immergés.

Dans une eau vierge de retenue très pauvre, comme toutes celles qui nous intéressent, le peuplement idéal par hectare semblerait devoir être :

32 Truites de 1 an pesant environ..... 1 k. 500

16 Truites de 2 ans pesant environ...... 2 kilos

8 Truites de 3 ans pesant environ...... 2 kilos

4 Truites de 4 ans pesant environ..... 2 kilos

2 Truites de 5 ans pesant environ..... 2 kilos

1 Truite de 6 ans pesant environ..... 2 kilos

11 k. 500

et nécessiterait donc un déversement annuel d'une cinquantaine d'estivaux.

Si les Ablettes ne pèsent qu'une douzaine de grammes en moyenne, c'est que le peuplement est complet et que la concurrence alimentaire joue à plein. Dans ce cas, il faudrait commencer par ne déverser qu'une vingtaine de truitelles par hectare chaque automne et, si les Truites pêchées ultérieurement ont un coefficient de forme satisfaisant, les déversements pourront être augmentés tant que certaines Truites pêchées ne seront pas manifestement maigres et que le pourcentage des reprises par les pêcheurs sera supérieur au dixième.

Cependant, le coefficient de forme peut dépendre de l'hérédité et du milieu indépendamment des ressources alimentaires et c'est, en fait, le grossissement annuel des truitelles déversées qu'il faudrait connaitre.

La meilleure méthode pour pouvoir connaître le grossissement des truitelles déversées est de les marquer.

Compte tenu de ce qu'il n'est pas utile de différencier entre elles les Truites déversées la même année, il suffira d'utiliser des marques caractérisées, au moyen d'une lettre pour chaque année. La lettre C a été utilisée pour les truitelles nées en 1958. 